\title{
PCR Primer Design for 16S rRNAs for Experimental Horizontal Gene Transfer Test in Escherichia coli
}

OPEN ACCESS

Edited by:

Diana Elizabeth Marco,

National Scientific Council

(CONICET), Argentina

Reviewed by:

Kei Kitahara,

Hokkaido University, Japan

Trevor Carlos Charles,

University of Waterloo, Canada

*Correspondence:

Kentaro Miyazak

miyazaki-kentaro@aist.go.jp

Specialty section:

This article was submitted to Microbiotechnology, Ecotoxicology

and Bioremediation,

a section of the journal Frontiers in Bioengineering and Biotechnology

Received: 19 December 2016 Accepted: 09 February 2017 Published: 28 February 2017

Citation:

Miyazaki K, Sato M and Tsukuda M (2017) PCR Primer Design for 16S rRNAs for Experimental Horizontal Gene Transfer Test in Escherichia coli.

Front. Bioeng. Biotechnol. 5:14. doi: 10.3389/fbioe.2017.00014

\begin{abstract}
Kentaro Miyazaki1,2*, Mitsuharu Sato ${ }^{1,2}$ and Miyuki Tsukuda ${ }^{1,2}$
${ }^{1}$ Department of Life Science and Biotechnology, Bioproduction Research Institute, National Institute of Advanced Industrial Science and Technology (AIST), Tsukuba, Ibaraki, Japan, ${ }^{2}$ Department of Computational Biology and Medical Sciences, Graduate School of Frontier Sciences, The University of Tokyo, Kashiwa, Chiba, Japan
\end{abstract}

We recently demonstrated that the Escherichia coli ribosome is robust enough to accommodate foreign 16S rRNAs from diverse gamma- and betaproteobacteria bacteria (Kitahara et al., 2012). Therein, we used the common universal primers Bac8f and UN1541r to obtain a nearly full-length gene. However, we noticed that these primers overlap variable sites at 19[A/C] and 1527[U/C] in Bac8f and UN1541r, respectively, and thus, the amplicon could contain mutations. This is problematic, particularly for the former site, because the 19th nucleotide pairs with the 916th nucleotide, which is a part of the "central pseudoknot" and is critical for function. Therefore, we mutationally investigated the role of the base pair using several 16S rRNAs from gamma- and betaproteobacteria. We found that both the native base pairs (gammaproteobacterial 19A-916U and betaproteobacterial 19C-916G) and the non-native 19A-916G pair retained function, whereas the non-native 19C-916U was defective 16S rRNAs. We next designed a new primer set, Bac1f and UN1542r, so that they do not overlap the potential mismatch sites. 16S rRNA amplicons obtained from the environmental metagenome using the new primer set were dominated by proteobacterial species ( $85 \%)$. Subsequent functional screening identified various $16 \mathrm{~S}$ rRNAs from proteobacteria, all of which contained native 19A-916U or 19C-916G base pairs. The primers developed in this study are thus advantageous for functional characterization of foreign 16S rRNA in E. coli with no artifacts.

Keywords: bacterial phylogeny, 16S rRNA, ribosome, horizontal gene transfer, molecular clock, functional complementation, metagenome, central pseudoknot

\section{INTRODUCTION}

The bacterial ribosome consists of 3 rRNA molecules and 54 proteins and plays a crucial role in translating mRNA-encoded information into proteins. Because of the structural complexity of the ribosome (Schuwirth et al., 2005), it is believed that each ribosomal component coevolves to maintain function (Jain et al., 1999). In particular, because the 16S and 23S rRNAs form the structural core of the ribosome (Schuwirth et al., 2005), they are believed to be least likely to experience horizontal gene transfer between species (Jain et al., 1999). On the basis of the species-specific nature of rRNA and their omnipresence in all bacteria, the rRNA genes, especially those for $16 \mathrm{~S}$ rRNA, have 
long been used as an "ultimate chronometer" (Woese, 1987) for phylogenetic classification of bacterial species (Lane et al., 1985; Woese, 1987).

Despite the apparent species-specific nature of $16 \mathrm{~S}$ rRNAs, we recently found that the Escherichia coli ribosome is able to accommodate foreign 16S rRNA (Kitahara et al., 2012). Namely, using $E$. coli $\Delta 7$, a null mutant of the $r r n$ (ribosomal RNA) operon, as a host strain, we have shown that various 16S rRNA genes, including those from a different phylogenetic class (i.e., betaproteobacteria), were able to complement growth. The lowest identity of functional $16 \mathrm{~S}$ rRNA gene to that of E. coli was as low as $80 \%$, implying that hundreds of simultaneous nucleotide changes are permitted in the maintenance of ribosome function. The basis for this high mutability is the conservation of the RNA secondary structures, which is consistent with a previous finding that $16 \mathrm{~S}$ rRNA is typically recognized by ribosomal proteins via salt bridges between phosphate oxygen atoms of the RNA backbone, but nucleotide bases are not strictly discriminated (Brodersen et al., 2002). Furthermore, insertion/deletion is allowed in some RNA helices (e.g., h6, 10, and 17) that are not involved in protein binding. Understanding the sequence and structural variations of $16 \mathrm{~S}$ rRNA that are accommodated in the E. coli ribosome should be helpful for our understanding of the evolution of rRNA and the sequence-structure-function relationships of the ribosome.

In our previous study, to PCR amplify foreign 16S rRNA genes, we used $\operatorname{Bac} 8 \mathrm{f}(\mathrm{A})$ or $\mathrm{Bac} 8 \mathrm{f}(\mathrm{C})$ [the most commonly used "Bac8f" (Eden et al., 1991)] for the forward primer and $\mathrm{UN1541r}(\mathrm{U})$ or UN1541r(C) for the reverse primer (Figure 1; oligonucleotide sequences summarized in Table 1) (Kitahara et al., 2012), which allowed amplification of a nearly full-length gene. These primers can cover the majority of bacterial $16 \mathrm{~S}$ rRNA genes and thus are commonly used for phylogenetic and/ or community analysis (Lane et al., 1985; Weisburg et al., 1991; Amann et al., 1995). However, we noticed that the amplicons obtained using the primer set contained mutations at certain frequencies, which could affect the functionality of in vivoreconstituted mutant ribosomes. In E. coli $16 \mathrm{~S}$ rRNA, nucleotides 17-19 pair with nucleotides 916-918 to form a short helix (h2) (Figure 2). The helix is involved in the formation of the "central pseudoknot," whose structure is highly conserved in both prokaryotes and eukaryotes. This unique structure is essential for translational initiation and is highly susceptible to point mutations (Brink et al., 1993; Dammel and Noller, 1993; Poot et al., 1998). Despite this structural conservation, however, the 19th nucleotide varies depending on the species, $19 \mathrm{~A}$ or $19 \mathrm{C}$, which pairs with $916 \mathrm{U}$ or $916 \mathrm{G}$, respectively (Figure 2). Thus, if $\operatorname{Bac} 8 \mathrm{f}(\mathrm{A})$ or $\operatorname{Bac} 8 \mathrm{f}(\mathrm{C})$ is used as a primer, there is a possibility of generating a mismatch between the 19th and 916th nucleotides. Similarly, the 1,527 th position is also variable ( $\mathrm{C}$ or $\mathrm{U})$ and can generate a mismatch in the amplicons (Figure 1B), although this site may not be involved in function. Thus, in our specific system for functional investigation of $16 \mathrm{~S}$ rRNAs, it is essential to develop a new primer set to avoid the introduction of artificial mutations. In addition, we need to

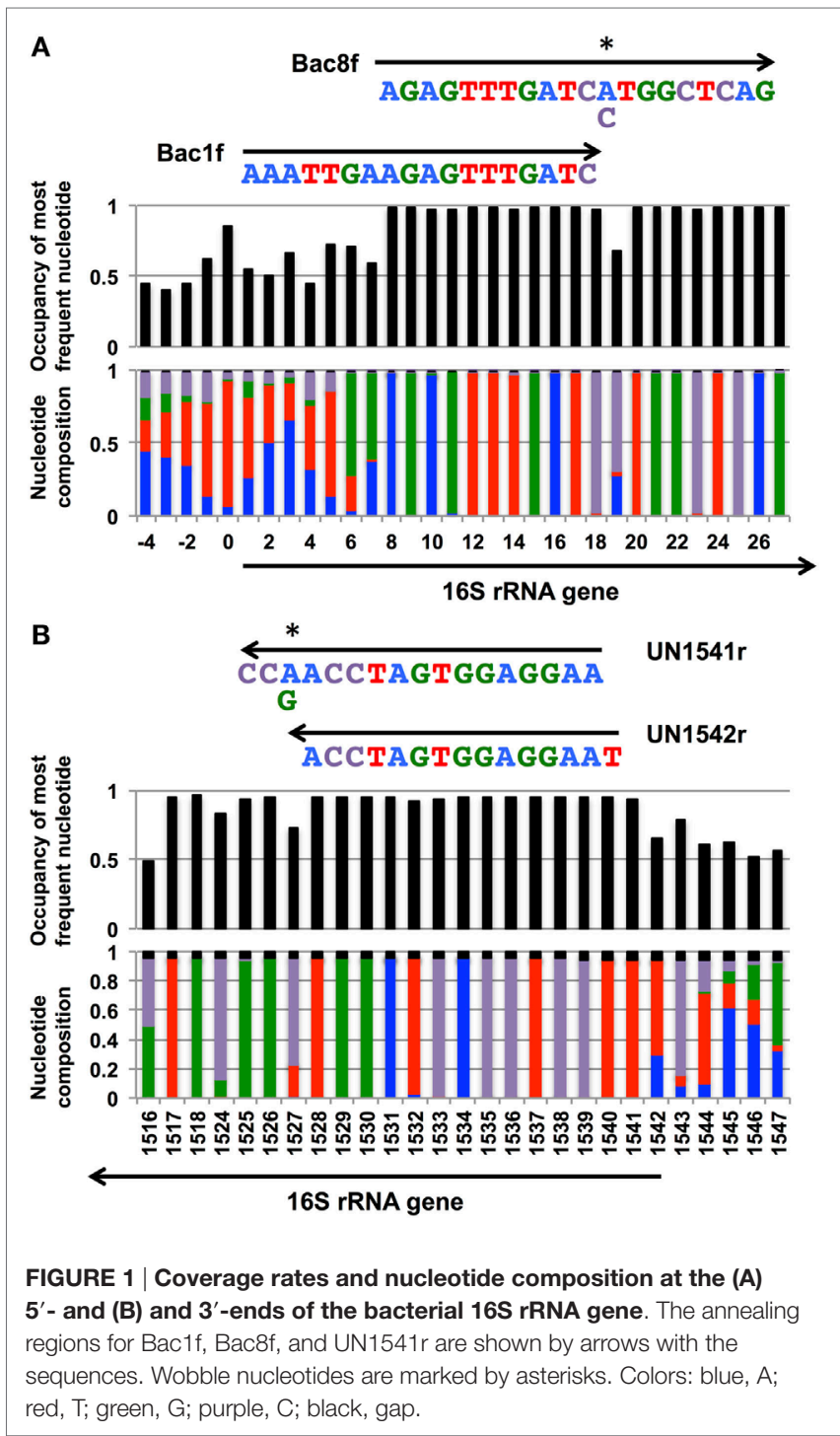

take the RNA processing issue into consideration. For proper processing of a precursor transcript into mature rRNAs (16S, 23S, and 5 S rRNAs), the processing sites (i.e., RNase cleavage sites) need to be similar to the E. coli sequences (Gutgsell and Jain, 2012).

Taking these points into consideration, we first evaluated the effects of mismatches between the 19th and 916th nucleotides. In addition to the native $19 \mathrm{~A}-916 \mathrm{U}$ and $19 \mathrm{C}-916 \mathrm{G}$ pairs, the nonnative 19A-916G pair retained function, whereas the non-native 19C-916U was detrimental. Second, we designed new primers, Baclf and UN1542r, which encompassed nucleotide positions 1-18 for Bac1f and 1542-1528 for UN1542r, so that they did not overlap the potential mismatch sites. These primers were used for PCR amplification of $16 S$ rRNA genes and the resultant library was functionally screened. DNA sequencing of the 16S rRNA genes in the functional clones confirmed the absence of unwanted mismatches in the functional genes. 
TABLE 1 | PCR primers used for amplification of 16S rRNA genes.

\begin{tabular}{|c|c|c|}
\hline Primer & Sequence & Reference \\
\hline Bac1f & 5'-AAATTGAAGAGTाTGATC-3' & This study \\
\hline $\operatorname{Bac} 8 f(A)^{a}$ & 5'-AGAGTITGATCA-TGGCTCAG-3' & Kitahara and Miyazaki (2011); Kitahara et al. (2012); Weisburg et al. (1991) \\
\hline $\operatorname{Bac} 8 f(C)^{a}$ & 5'-AGAGTTTGATCĒTGGCTCAG-3' & Kitahara and Miyazaki (2011); Kitahara et al. (2012); Weisburg et al. (1991) \\
\hline UN1542r & 5'-TAAGGAGGTGĀTCCA-3' & This study \\
\hline UN1541r(U) ${ }^{a}$ & 5'-AAGGAGGTGATCCAACC-3' & Kitahara and Miyazaki (2011); Kitahara et al. (2012) \\
\hline UN1541r(C) $)^{\mathrm{a}}$ & 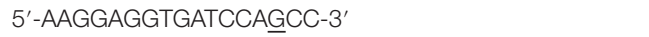 & Kitahara and Miyazaki (2011); Kitahara et al. (2012); Weisburg et al. (1991) \\
\hline Bac1r & 5'-GATCAAACTCTTCAĀTTAAAAGTTGACGCTCAAAG-3' & This study \\
\hline $\operatorname{Bac} 8 \mathrm{r}(\mathrm{A})$ & 5'-CTGAGCCATGATCAAACTCTTC-3' & Kitahara and Miyazaki (2011); Kitahara et al. (2012) \\
\hline $\operatorname{Bac} 8 \mathrm{r}(\mathrm{C})$ & 5'-CTGAGCCAGGATCAAACTCTTC-3' & This study \\
\hline UN1542f & 5'-TGGATCACCTCCTTACCTTAAAGAAGCGT-3' & This study \\
\hline
\end{tabular}

aUnderlined nucleotides are the sites that can generate a mismatch.

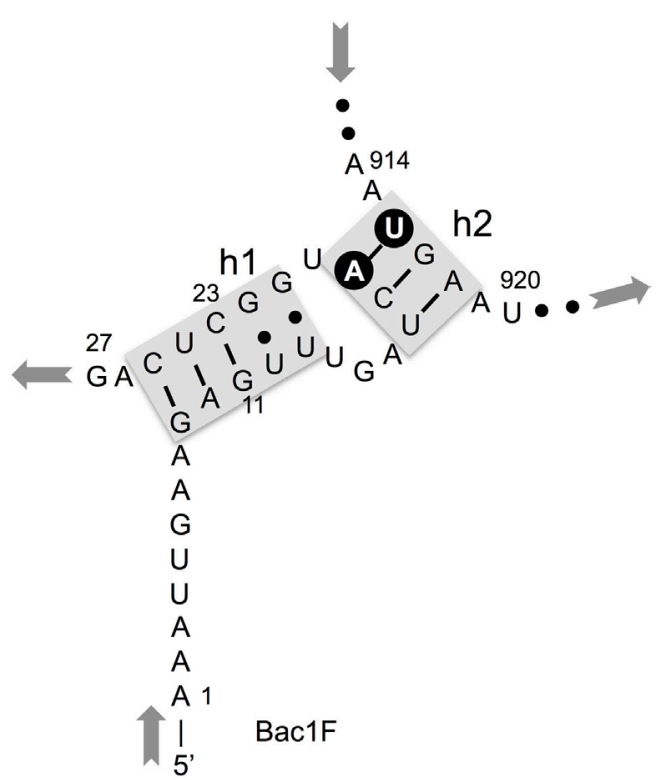

FIGURE 2 | Central pseudoknot structure in Escherichia coli 16S rRNA. Nucleotide numbers 19 and 916 are labeled in white letters in a black circle.

\section{MATERIALS AND METHODS}

\section{Reagents}

KOD-Plus-Neo and KOD FX-Neo DNA polymerases were purchased from Toyobo (Osaka, Japan). Trimethoprim (Tmp), ampicillin (Amp), kanamycin (Km), and sucrose (Suc) were purchased from Wako Pure Chemicals (Tokyo, Japan). Zeocin ${ }^{\mathrm{TM}}$ (Zeo) and the In-Fusion Cloning Kit were purchased from Invitrogen (Carlsbad, CA, USA). Lennox LB medium [1\% (w/v) tryptone, $0.5 \%(\mathrm{w} / \mathrm{v})$ yeast extract, $0.5 \%(\mathrm{w} / \mathrm{v})$ $\mathrm{NaCl}$ ] was purchased from Merck(Tokyo, Japan). The Extrap SoilDNA Kit Plus ver. 2 was purchased from J-Bio21 (Tsukuba, Japan). FastDNA Kit was purchased from BIO101 (La Jolla, CA, USA). Oligonucleotide primers (Table 1) were purchased from Sigma (Hokkaido, Japan).

\section{Bacterial Strains and Culture Conditions}

The following bacterial strains were purchased from the Biological Resource Center (NBRC), National Institute of Technology and
Evaluation, Japan: Serratia ficaria (NBRC 102596), Caldimonas manganoxidans (NBRC 16448), Hydrogenophaga flava (NBRC 102514), Hydrogenophilus thermoluteolus (NBRC 14978), Oxalicibacterium horti (NBRC 13594), Oligella urethralis (NBRC 14589), and Ralstonia pickettii (NBRC 102503). Burkholderia sacchari was a laboratory stock. Competent E. coli JM109 cells were purchased from RBC Bioscience (Taipei, Taiwan). Antibiotics were added when necessary at the following concentrations: Tmp, $10 \mu \mathrm{g} / \mathrm{ml} ;$ Amp, $100 \mu \mathrm{g} / \mathrm{ml}$ Km, $25 \mu \mathrm{g} / \mathrm{ml}$; and Zeo, $50 \mu \mathrm{g} / \mathrm{ml}$. Agar $(1.5 \%[\mathrm{w} / \mathrm{v}])$ was added to solidify the medium. Suc was added at $5 \%(\mathrm{w} / \mathrm{v})$ for counterselection purposes when necessary.

\section{Genomic DNA Purification, PCR Amplification of 16S rRNA Genes, and Library Construction}

Genomic DNA of bacterial isolates was purified using the FastDNA Kit. The Nitrosomonas europaea was a gift from Dr. Naohiro Noda (AIST, Japan). Environmental metagenomic DNA (soils, fermented products, and seawater) was purified using the Extrap Soil DNA Kit Plus ver. 2. The 16S rRNA genes were amplified by PCR using a set of primers, Baclf, Bac8f(A), or Bac8f(C) and UN1542r. The reaction mixture contained $100 \mathrm{ng}$ of template DNA (bacterial genome or environmental metagenome), $1 \times$ PCR buffer, $0.4 \mathrm{mM}$ each of dNTPs, $0.25 \mu \mathrm{M}$ each of primers and $1 \mathrm{U}$ of KOD FX-Neo DNA polymerase in a total volume of $50 \mu \mathrm{l}$. The mixture was heated at $94^{\circ} \mathrm{C}$ for $2 \mathrm{~min}$ and subjected to 30 rounds of thermal cycling at $98^{\circ} \mathrm{C}$ for $10 \mathrm{~s}, 48^{\circ} \mathrm{C}$ for $30 \mathrm{~s}$ for Bac $1 \mathrm{f}$ or $57^{\circ} \mathrm{C}$ for $30 \mathrm{~s}$ for $\operatorname{Bac} 8 f(A)$ and $\operatorname{Bac} 8 f(C)$, and $68^{\circ} \mathrm{C}$ for $1.5 \mathrm{~min}$ and final incubation at $68^{\circ} \mathrm{C}$ for $5 \mathrm{~min}$. The amplicon was separated by agarose gel $(0.8 \%[\mathrm{w} / \mathrm{v}])$ electrophoresis; a single band was excised from the gel, purified and dissolved in $30 \mu \mathrm{l}$ of water.

An expression vector for the 16S rRNA gene was modified from pRB103 (Kitahara and Suzuki, 2009; Kitahara et al., 2012) by deleting the genes for tRNA, 23S rRNA, and 5S rRNA, replacing the antibiotic selection marker from Zeo to Tmp and replication origin from pSC101 to p15A. The resultant plasmid was named pMS205aTp1 (map illustrated in Figure S1A in Supplementary Material). The entire vector (without the 16S rRNA gene) was PCR-amplified using the primer set Bac1R, Bac8r(A), or Bac8r $(\mathrm{C})$ and UN1542f. The PCR mixture contained $1 \times$ PCR buffer, $0.2 \mathrm{mM}$ each of dNTPs, $1.5 \mathrm{mM} \mathrm{MgSO}_{4}, 0.25 \mu \mathrm{M}$ each of primers, $10 \mathrm{ng}$ of pMS205aTp1, and $1 \mathrm{U}$ of KOD-Neo-DNA 
polymerase in a total volume of $50 \mu \mathrm{l}$. The mixture was heated at $94^{\circ} \mathrm{C}$ for $2 \mathrm{~min}$ and subjected to 25 cycles at $94^{\circ} \mathrm{C}$ for $10 \mathrm{~s}, 60^{\circ} \mathrm{C}$ for $30 \mathrm{~s}$, and $68^{\circ} \mathrm{C}$ for $2.5 \mathrm{~min}$, followed by a final incubation at $68^{\circ} \mathrm{C}$ for $5 \mathrm{~min}$. The products were treated with $\mathrm{DpnI}\left(10 \mathrm{U}, 37^{\circ} \mathrm{C}\right.$, $6 \mathrm{~h}$ ), gel-purified and dissolved in $30 \mu \mathrm{l}$ of water.

The 16S rRNA gene (ca., $200 \mathrm{ng}$ ) and the linearized pMS205aTp1 (ca., $200 \mathrm{ng}$ ) fragments were combined and ligated using the In-Fusion Cloning Kit in a total volume of $10 \mu \mathrm{l}$. After incubation at $50^{\circ} \mathrm{C}$ for $1 \mathrm{~h}$, the reaction products $(2 \mu \mathrm{l})$ were introduced into competent $E$. coli JM109 cells (100 $\mu \mathrm{l})$ and grown on $\mathrm{LB} / \mathrm{Tmp}$ agar plates at $37^{\circ} \mathrm{C}$ overnight. Some of the colonies were randomly picked and used for sequence determination for phylogenetic analysis. Rest of the colonies were combined and plasmids were extracted to yield a library.

\section{Functional Screening of 16S rRNA Genes}

Escherichia coli MY201 $\mathrm{rna}^{-}$is a derivative of E. coli $\Delta 7 \mathrm{rna}^{-}$ (Kitahara and Suzuki, 2009), which contains the growth rescue plasmid pMY201 (modified from pRB101 by substituting the pSC101 ori to p15A ori, Figure S1B in Supplementary Material) and pML103 $\Delta$ (expression plasmid for 23S rRNA, 5S rRNA, and tRNAs, created by deleting the 16S rRNA gene from pRB103, map illustrated in Figure S1C in Supplementary Material). Competent MY201 cells were transformed with a pMS205aTp1 library and grown on $\mathrm{LB} / \mathrm{Km} / \mathrm{Zeo} / \mathrm{Tmp}$ agar plates at $37^{\circ} \mathrm{C}$ for overnight. Colonies were collected, mixed in $1 \mathrm{ml}$ of $\mathrm{LB}$ broth, vigorously vortexed, appropriately diluted, and spread over $\mathrm{LB} / \mathrm{Km} / \mathrm{Zeo} /$ $\mathrm{Tmp} /$ Suc agar plates. Some of the colonies were randomly picked and used for sequence determination for phylogenetic analysis. Rest of the colonies grown on the plates were collected and used for further studies.

\section{Growth Assay}

Mutant E. coli strains were grown in $1 \mathrm{ml}$ of $\mathrm{LB} / \mathrm{Km} / \mathrm{Zeo} / \mathrm{Tmp} /$ Suc broth in a 96 deep-well plate. The plate was incubated at $37^{\circ} \mathrm{C}$ with vigorous agitation (1,200 rpm) in an MBR-024 microplate shaker (Taitec, Saitama, Japan). After $14 \mathrm{~h}, 1 \mu \mathrm{l}$ of the culture was transferred to a fresh $\mathrm{LB} / \mathrm{Km} / \mathrm{Zeo} / \mathrm{Tmp} / \mathrm{Suc}$ broth $(1 \mathrm{ml})$ in 96-well plate and grown at various temperatures $\left(30,37\right.$, or $\left.42^{\circ} \mathrm{C}\right)$ with vigorous agitation $(1,200 \mathrm{rpm})$. After $14 \mathrm{~h}, 200 \mu \mathrm{l}$ of the culture was transferred to a 96 -well plate and $\mathrm{OD}_{600}$ was measured.

\section{DNA Sequencing and BLAST Search}

DNA sequencing was carried out using the Sanger method with an Applied Biosystems (Foster City, CA, USA) automatic DNA sequencer (ABI PRISM 3130xl Genetic Analyzer) and an Applied Biosystems BigDye (ver. 3.1) kit. Blast search (Altschul et al., 1990) was carried out using the NCBI nucleotide database "16S rRNA sequences (Bacteria and Archaea)" with the program selection optimized for "Highly similar sequences (megablast)."

\section{Dataset and Sequence Alignment of $16 \mathrm{~S}$ rRNA Genes}

All 16S rRNA gene sequences (plus 50 additional nucleotides at the $5^{\prime}$ and $3^{\prime}$ ends) were retrieved from the genomic sequences in the NCBI database (as of August 2014) (Table 2). A total of 9,624 genes were identified in 2,476 genomes of 23 phyla. Multiple
TABLE 2 | List of 16S rRNA genes retrieved from the NCBI database. ${ }^{a}$

\begin{tabular}{|c|c|c|}
\hline Phylum or group & $\begin{array}{c}\text { Number of } \\
\text { genomes }\end{array}$ & $\begin{array}{c}\text { Number of } \\
\text { 16S rRNA } \\
\text { genes }\end{array}$ \\
\hline Actinobacteria & 268 & 833 \\
\hline Aquificae & 13 & 27 \\
\hline Bacteroidetes-Chlorobi group & 99 & 287 \\
\hline Caldiserica & 1 & 1 \\
\hline Chlamydiae-Verrucomicrobia group & 112 & 200 \\
\hline Chloroflexi & 20 & 34 \\
\hline Chrysiogenetes & 1 & 3 \\
\hline Deferribacteres & 4 & 7 \\
\hline Cyanobacteria & 75 & 168 \\
\hline Dictyoglomi & 2 & 4 \\
\hline Deinococcus-Thermus & 22 & 51 \\
\hline Elusimicrobia & 1 & 1 \\
\hline Fibrobacteres-Acidobacteria group & 7 & 12 \\
\hline Firmicutes & 532 & 3,304 \\
\hline Fusobacteria & 9 & 38 \\
\hline Nitrospirae & 4 & 7 \\
\hline Planctomycetes & 7 & 18 \\
\hline Proteobacteria & 1,144 & 4,369 \\
\hline Spirochaetes & 58 & 106 \\
\hline Synergistetes & 4 & 11 \\
\hline Tenericutes & 74 & 111 \\
\hline Thermodesulfobacteria & 2 & 3 \\
\hline Thermotogae & 17 & 29 \\
\hline Total & 2,476 & 9,624 \\
\hline
\end{tabular}

${ }^{a} A l l 16 S$ rRNA gene sequences were extracted from all available genomic sequences in NCBI database on August 1, 2014

sequence alignment of these genes was performed using the MAFFT v7 program (Katoh and Standley, 2013).

\section{Nucleotide Sequence Accession Numbers}

The nucleotide sequences for 16S rRNA gene have been deposited in GenBank/EMBL/DDBJ under the accession numbers LC213146-LC213207, LC213207-LC213252, and LC213253-LC213296.

\section{RESULTS AND DISCUSSION}

\section{Primer Design}

Nucleotide composition around the $5^{\prime}$ - and $3^{\prime}$-end regions of all bacterial 16S rRNA genes (Table 2) is shown in Figure 1. For the $5^{\prime}$-end, the sequence surrounding the 19th nucleotide, particularly from the 8 th to 27 th, is highly conserved (Figure 1A), which corresponds to the Bac8f primer-binding site. The Bac8f primer covers $97 \%$ of bacterial $16 \mathrm{~S}$ rRNA sequences (Figure 1A), confirming the appropriateness of the primer for phylogenetic/ community analysis (Lane et al., 1985; Amann et al., 1995). However, due to the presence of a potential mismatch site at the 19th nucleotide position, this is not appropriate for our specific purpose (i.e., functional analysis), and thus, we designed a new primer Bac1f, which encompasses the 1st to 18th nucleotides. Although the very beginning of the sequence (from first to seventh nucleotides) is highly variable among all bacteria (Figure 1A), the region is critical for RNA processing (Gutgsell and Jain, 2012), so we strictly followed the E. coli sequence for this site. 
We next checked the coverage rate of the Baclf primer for each phylum (Figure S2 in Supplementary Material). As described above, the $5^{\prime}$ end of the 16S rRNA sequence varies among bacteria (Figure 1A). Nevertheless, Baclf showed relatively high specificity to some bacterial $16 \mathrm{~S}$ rRNAs that included Bacteroidetes-Chlorobi (Figure S2B in Supplementary Material), Chlamydiae-Verrucomicrobia (Figure S2C in Supplementary Material), and Proteobacteria (Figure S2G in Supplementary Material). In our previous study (Kitahara et al., 2012), no functional 16S rRNAs were obtained from phyla other than proteobacteria. Thus, in practice, although the Baclf primer has some bias to specific phylogenetic groups, this bias is advantageous to enriching libraries with a potentially functional fraction and to reducing background.

Figure S3 in Supplementary Material summarizes the coverage rate of the Baclf primer for each class of proteobacteria. Overall, there is a slight preference for alpha-, beta-, and gamma-classes of proteobacteria, and the delta-epsilon class has a larger number of potential mismatches.

\section{Effects of Non-Natural Base Pairing between the 19th and 916th Nucleotides on Ribosomal Activity}

To investigate how non-natural base pairing between the 19th and 916th nucleotides affects ribosomal activity, we used 16S rRNA genes from the following bacteria: gammaproteobacterial E. coli (Eco) and S. ficaria (Sfi) and betaproteobacterial B. sacchari (Bsa), C. manganoxidans (Cma), H. flava (Hfl), H. thermoluteolus (Hth), N. europaea (Neu), O. horti (Oho), O. urethralis (Our), and R. pickettii (Rpi).

PCR amplification was carried out using three types of forward primers: Bac1f, Bac8f(A), or Bac8f(C); UN1542r was used as a common reverse primer. A specifically amplified fragment was then cloned back into pMS205aTp1. After confirming the sequence of the entire $16 \mathrm{~S}$ rRNA gene, the resultant plasmid was transferred into $E$. coli MY201 $r n a^{-}$( $\Delta 7$ strain). After sacB-based counterselection to eliminate rescue plasmids expressing $E$. coli $16 \mathrm{~S}$ rRNA, all the clones were successfully obtained at $37^{\circ} \mathrm{C}$, implying that mutation in the 19th-916th base pair is more or less permissive under this condition.

We next examined the growth properties of each mutant at various temperatures $\left(30^{\circ} \mathrm{C}, 37^{\circ} \mathrm{C}\right.$, and $\left.42^{\circ} \mathrm{C}\right)$. Figure $3 \mathrm{~A}$ illustrates $\mathrm{OD}_{600}$ after growth for $14 \mathrm{~h}$. In general, for the native base pairs (19A-916U for gammaproteobacterial and 19C-916G for betaproteobacterial $16 \mathrm{~S}$ rRNAs) all clones had higher $\mathrm{OD}_{600}$ at $37^{\circ} \mathrm{C}$ than at $30^{\circ} \mathrm{C}$. For the clones carrying non-native pairs (Figure 3B, 19C-916U for gammaproteobacterial and 19A-916G for betaproteobacterial 16S rRNAs), no growth perturbation was observed for betaproteobacterial clones and they appeared to gain a broadened temperature optimum; final $\mathrm{OD}_{600}$ shifted upward at $30^{\circ} \mathrm{C}$. In contrast, gammaproteobacterial clones (Eco and Sfi) showed greatly reduced $\mathrm{OD}_{600}$ values at all temperatures. Virtually no growth was observed at $30^{\circ} \mathrm{C}$, indicating the appearance of a cold-sensitive (or heat-tolerant) phenotype.

Poot et al. (1998) have analyzed the role of h2 through mutagenesis. Using E. coli $16 \mathrm{~S}$ rRNA as a template, they introduced a point
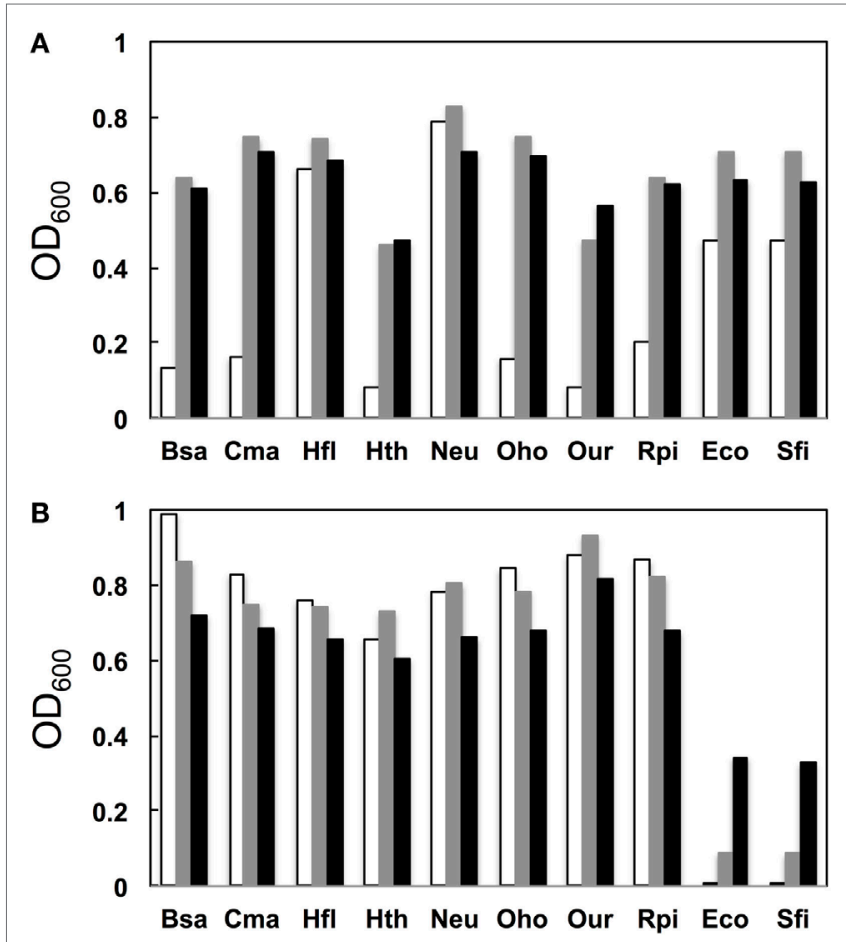

FIGURE 3 | Growth profile. (A) Native base pairs between nucleotides 19 and 916 [19C-916G for betaproteobacterial Burkholderia sacchari (Bsa), Caldimonas manganoxidans (Cma), Hydrogenophaga flava $(\mathrm{Hfl})$,

Hydrogenophilus thermoluteolus ( $\mathrm{Hth}$ ), Nitrosomonas europaea (Neu), Oxalicibacterium horti (Oho), Oligella urethralis (Our), and Ralstonia pickettii (Rpi) and 19A-916U for gammaproteobacterial Escherichia coli (Eco) and Serratia ficaria (Sfi)] and (B) non-native base pairs between nucleotides 19 and 916 (19A-916G for betaproteobacterial Bsa, Cma, Hfl, Hth, Neu, Oho, Our, and Rpi and 19C-916U for gammaproteobacterial Eco and Sfi). y axis represents $\mathrm{OD}_{600}$ after cultivation at various temperatures $\left(30^{\circ} \mathrm{C}\right.$, open bars; $37^{\circ} \mathrm{C}$, shaded bars; $42^{\circ} \mathrm{C}$, solid bars) for $14 \mathrm{~h}$.

mutation to alter the native $19 \mathrm{~A}-916 \mathrm{U}$ base pair to $19 \mathrm{~A}-916 \mathrm{G}$, 19C-916G, and 19C-916U. They used the mutant ribosome in an in vitro translational assay (at $42^{\circ} \mathrm{C}$ ) and found that the former two mutants retained nearly full activity (>80\%), whereas 19C-916U had much reduced (30\%) activity. Although the assay systems are different, the general conclusion of both their study and ours is that $19 \mathrm{C}-916 \mathrm{U}$ is defective.

\section{Metagenomic Screening for Functional $16 S$ rRNA Genes in $E$. coli}

We next used environmental metagenomes as a source for $16 \mathrm{~S}$ rRNA genes. For all primer sets, specific amplification was obtained (Figure S4 in Supplementary Material). To investigate sequence diversity, the cloned genes were phylogenetically characterized. As shown in Figure 4, when Baclf was used as a forward primer, the gene was dominated by proteobacterial $16 \mathrm{~S}$ rRNAs [ 84\% (32/38)], but was much less for those obtained using the $\operatorname{Bac} 8 \mathrm{f}(\mathrm{A})$ and Bac $8 f(C)$ primers [ 56\% (25/45) and $73 \%$ (35/48), respectively].

The genes were then subjected to functional screening. Functional 16S rRNA genes were collected, and their microbial 


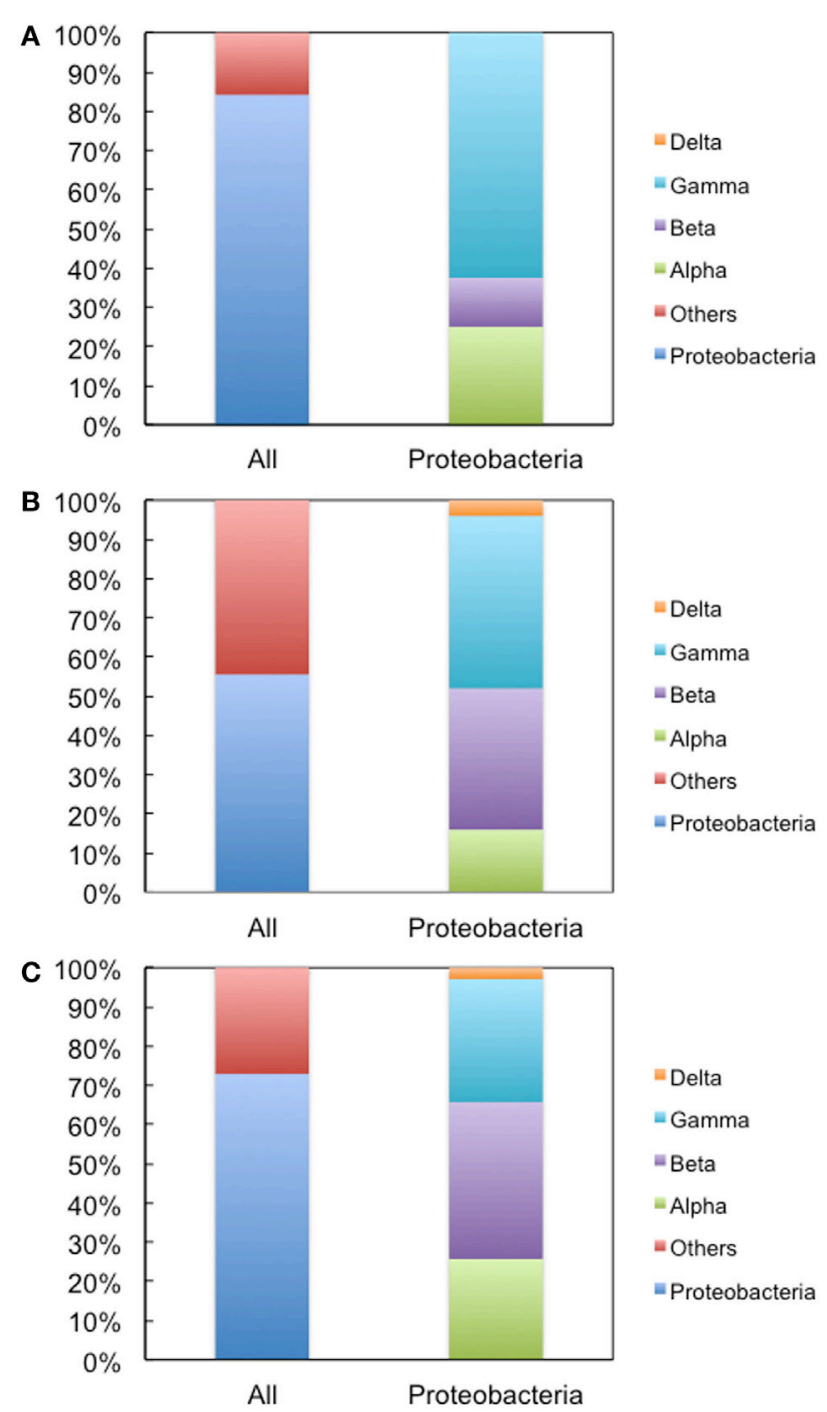

FIGURE 4 | Phylogenetic analysis of 16S rRNA genes cloned in pMS205aTp1 (before functional selection) amplicons. The forward primers used were (A) Bac1f, (B) Bac8f(A), and (C) Bac8f(C). "All" is the classification based on phylum and "Proteobacteria" is the class composition in the proteobacterial phylum.

origins and the base pair patterns between the 19th and 916th nucleotides were investigated. As shown in Table 3, when Baclf was used, $85 \%$ (52/61) of functional $16 \mathrm{~S}$ rRNAs were from gammaproteobacteria and the rest were from betaproteobacteria. Phylogenetic tree of these sequences is illustrated in Figure S5A in Supplementary Material. Base pair patterns were 78\% 19A-916U $(48 / 61)$ and $22 \%(13 / 61)$ 19C-916G. It is noteworthy that no artificially shuffled base pairs (A-G and $\mathrm{C}-\mathrm{U}$ ) were observed, implying that the newly designed primers did not introduce non-native base pairs and are adequate for functional studies. There are some mismatches between proteobacterial 16S rRNA and Bac1f sequences (Figure S3 in Supplementary Material), but in practice, we succeeded in retrieving various functional genes, suggesting that the mismatches at the $5^{\prime}$-end can affect annealing efficiency, but still remain effective for amplification. The lack of
TABLE 3 | Sequence analysis of functional 16S rRNA genes in Escherichia coli $\Delta 7$ retrieved from the metagenome.

\begin{tabular}{lrrr}
\hline & Bac1f & Bac8f(A) & Bac8f(C) \\
\hline Predicted microbial origins & 52 & 39 & 13 \\
Gammaproteobacteria & 9 & 6 & 29 \\
Betaproteobacteria & 0 & 0 & 2 \\
Deltaproteobacteria & 61 & 45 & 44 \\
Total & & & \\
Base pairs between the 19th and 916 th nucleotides & & 0 \\
A-G & 0 & 21 & 1 \\
A-U & 48 & 23 & 42 \\
C-G & 13 & 1 & 1 \\
C-U & 0 & 0 & 44 \\
Total & 61 & 45 & \\
\hline
\end{tabular}

any alphaproteobacterial 16S rRNAs in our functional 16S rRNA collection may be due to functional incompatibility in $E$. coli.

When $\operatorname{Bac} 8 \mathrm{f}(\mathrm{A})$ was used as a forward primer, 87\% (39/45) of functional $16 \mathrm{~S}$ rRNAs were of gammaproteobacteria and the rest $(13 \% ; 6 / 45)$ were of betaproteobacteria. Phylogenetic tree of these sequences is illustrated in Figure S5B in Supplementary Material. When the base pair patterns were investigated, noncanonical base pairs were frequently observed. Approximately half $(21 / 45)$ of the sequences contained the 19A-916G base pair, which may have resulted from the mis-annealing of the primer to template 16S rRNA genes containing 19C and 916G. Because this artificial base pair is permissive (or even encouraged) under normal growth conditions (Figure 3B), it is reasonable to find these species; simple PCR conditioning may not easily remove these mis-annealed products.

When Bac8f(C) was used as a forward primer, 66\% (29/44) of functional 16S rRNAs were of betaproteobacteria, 30\% (13/44) were of gammaproteobacteria, and 5\% (2/44) were of deltaproteobacteria. Phylogenetic tree of these sequences is illustrated in Figure S5C in Supplementary Material. In this library, nonnatural base pair patterns were rarely observed. Most were of the 19C-916G base pair and the non-native 19C-916U base pair was found in one clone (closest relative was gammaproteobacterial Rahnella aquatilis, NR_074921). This low-level occurrence of the 19C-916U base pair agreed with the detrimental (yet still nonlethal) effect of the pair on cell growth (Figure 3B).

In conclusion, we developed a new primer set, Baclf and UN1542r, for the functional study of $16 \mathrm{~S}$ rRNAs in E. coli. The effective utilization of the primers was demonstrated by retrieval of a range of functional $16 \mathrm{~S}$ rRNAs from the proteobacterial lineage, all of which contained a native base pair between the 19th and 916th nucleotides.

\section{AUTHOR CONTRIBUTIONS}

KM, MS, and MT designed the study, conducted the data analysis, and wrote the manuscript.

\section{ACKNOWLEDGMENTS}

The authors thank Dr. Naohiro Noda (AIST, Japan) for the genomic DNA of Nitrosomonas europaea. This work was 
supported by a JSPS Grant-in-Aid for Scientific Research (B) (Grant 26292048 to KM), Grant-in-Aid for Scientific Research on Innovative Areas (Grant 26670219 to KM), Grant-in-Aid for Challenging Exploratory Research (Grant 15H01072 to KM), and Grant-in-Aid for JSPS Fellows 26-7760 (to MT).

\section{REFERENCES}

Altschul, S. F., Gish, W., Miller, W., Myers, E. W., and Lipman, D. J. (1990). Basic local alignment search tool. J. Mol. Biol. 215, 403-410. doi:10.1016/ s0022-2836(05)80360-2

Amann, R. I., Ludwig, W., and Schleifer, K. H. (1995). Phylogenetic identification and in situ detection of individual microbial cells without cultivation. Microbiol. Rev. 59, 143-169.

Brink, M. F., Verbeet, M. P., and De Boer, H. A. (1993). Formation of the central pseudoknot in $16 \mathrm{~S}$ rRNA is essential for initiation of translation. EMBO J. 12, 3987-3996.

Brodersen, D. E., Clemons, W. M. Jr., Carter, A. P., Wimberly, B. T., and Ramakrishnan, V. (2002). Crystal structure of the $30 \mathrm{~S}$ ribosomal subunit from Thermus thermophilus: structure of the proteins and their interactions with 16 S RNA. J. Mol. Biol. 316, 725-768. doi:10.1006/jmbi.2001.5359

Dammel, C. S., and Noller, H. F. (1993). A cold-sensitive mutation in 16S rRNA provides evidence for helical switching in ribosome assembly. Genes Dev. 7, 660-670. doi:10.1101/gad.7.4.660

Eden, P. A., Schmidt, T. M., Blakemore, R. P., and Pace, N. R. (1991). Phylogenetic analysis of Aquaspirillum magnetotacticum using polymerase chain reaction-amplified 16S rRNA-specific DNA. Int. J. Syst. Bacteriol. 41, 324-325. doi:10.1099/00207713-41-2-324

Gutgsell, N. S., and Jain, C. (2012). Gateway role for rRNA precursors in ribosome assembly. J. Bacteriol. 194, 6875-6882. doi:10.1128/jb.01467-12

Jain, R., Rivera, M. C., and Lake, J. A. (1999). Horizontal gene transfer among genomes: the complexity hypothesis. Proc. Natl. Acad. Sci. U.S.A. 96, 3801-3806. doi:10.1073/pnas.96.7.3801

Katoh, K., and Standley, D. M. (2013). MAFFT multiple sequence alignment software version 7: improvements in performance and usability. Mol. Biol. Evol. 30, 772-780. doi:10.1093/molbev/mst010

Kitahara, K., and Suzuki, T. (2009). The ordered transcription of RNA domains is not essential for ribosome biogenesis in Escherichia coli. Mol. Cell 34, 760-766. doi:10.1016/j.molcel.2009.05.014

\section{SUPPLEMENTARY MATERIAL}

The Supplementary Material for this article can be found online at http://journal.frontiersin.org/article/10.3389/fbioe.2017.00014/ full\#supplementary-material.

Kitahara, K., and Miyazaki, K. (2011). Specific inhibition of bacterial RNase T2 by helix 41 of $16 \mathrm{~S}$ ribosomal RNA. Nat. Commun. 2, 549. doi:10.1038/ ncomms 1553

Kitahara, K., Yasutake, Y., and Miyazaki, K. (2012). Mutational robustness of 16S ribosomal RNA, shown by experimental horizontal gene transfer in Escherichia coli. Proc. Natl. Acad. Sci. U.S.A. 109, 19220-19225. doi:10.1073/ pnas.1213609109

Lane, D. J., Pace, B., Olsen, G. J., Stahl, D. A., Sogin, M. L., and Pace, N. R. (1985). Rapid determination of $16 \mathrm{~S}$ ribosomal RNA sequences for phylogenetic analyses. Proc. Natl. Acad. Sci. U.S.A. 82, 6955-6959. doi:10.1073/pnas.82.20. 6955

Poot, R. A., Van Den Worm, S. H., Pleij, C. W., and Van Duin, J. (1998). Base complementarity in helix 2 of the central pseudoknot in 16S rRNA is essential for ribosome functioning. Nucleic Acids Res. 26, 549-553. doi:10.1093/nar/26.2.549

Schuwirth, B. S., Borovinskaya, M. A., Hau, C. W., Zhang, W., Vila-Sanjurjo, A., Holton, J. M., et al. (2005). Structures of the bacterial ribosome at $3.5 \AA$ resolution. Science 310, 827-834. doi:10.1126/science.1117230

Weisburg, W. G., Barns, S. M., Pelletier, D. A., and Lane, D. J. (1991). 16S ribosomal DNA amplification for phylogenetic study. J. Bacteriol. 173, 697-703. doi:10.1128/jb.173.2.697-703.1991

Woese, C. R. (1987). Bacterial evolution. Microbiol. Rev. 51, 221-271.

Conflict of Interest Statement: The authors declare that the research was conducted in the absence of any commercial or financial relationships that could be construed as a potential conflict of interest.

Copyright (c) 2017 Miyazaki, Sato and Tsukuda. This is an open-access article distributed under the terms of the Creative Commons Attribution License (CC BY). The use, distribution or reproduction in other forums is permitted, provided the original author(s) or licensor are credited and that the original publication in this journal is cited, in accordance with accepted academic practice. No use, distribution or reproduction is permitted which does not comply with these terms. 